\title{
Strategy of Starting Sensorless BLDCM with Inductance Method and EMF Integration
}

\author{
Wang Dafang, ${ }^{1}$ Qi Ji, ${ }^{1}$ Zhu Cheng, ${ }^{2}$ Liao Jiangmin, ${ }^{1}$ and Yuan Yechen ${ }^{1}$ \\ ${ }^{1}$ School of Automotive Engineering, Harbin Institute of Technology, Weihai 264209, China \\ ${ }^{2}$ China Automotive Technology \& Research Center, Tianjin 300300, China \\ Correspondence should be addressed to Wang Dafang; wdflcjl@163.com
}

Received 13 October 2013; Accepted 2 November 2013

Academic Editor: Hamid Reza Karimi

Copyright (C) 2013 Wang Dafang et al. This is an open access article distributed under the Creative Commons Attribution License, which permits unrestricted use, distribution, and reproduction in any medium, provided the original work is properly cited.

\begin{abstract}
In, conventional 3-stage start-up method of sensorless brushless direct current motor (BLDCM), the rotor is likely to jitter because rotor position cannot be obtained, and the motor is apt to lose step when it starts with load. These defects limit its use in engineering applications. In order to achieve smooth start in specific direction and guarantee start-up success rate with load, a start-up method based on improved inductance method and electromotive force (EMF) integration is proposed applying different voltage vectors according to rotor position interval judged by inductance method and determining integrator start-up time according to rotor initial position and the EMF. Experiments show that the method guarantees smooth acceleration and increases start-up success rate with load.
\end{abstract}

\section{Introduction}

BLDCM drive system has a wide range of applications in automobiles, such as the electric power steering system, the main drive system of small electric vehicle, and the new fuel pump systems of the traditional vehicle, and it is developing toward sensorless control. Comparing sensorless control with a position sensor control method, it has advantages of compact structure and high reliability because of saving installation space and reducing the sensor signal wires. So in recent years, the sensorless control of BLDCM has become a hot spot. At present, the main disadvantage limiting the application of sensorless BLDCM is that it is rather hard to start it directly. Now, a 3-stage start-up method [1] is a successful approach used in the start-up stage. The three stages are rotor preposition, acceleration, and switching. Among them, the rotor preposition mainly involves conducting the two phases with each other and forcing the rotor to rotate to the specified location. This method can be applied in cases where accuracy requirement is not high. For the sensorless BLDCM drive system used in the automobile, the size of the load cannot be predicted and the rotor is prevented from running freely at start-up. The existing start-up method cannot fit the requirement and also cannot guarantee the start-up success rate under load $[2,3]$.

Over the years, in order to solve this problem, many start-up methods used for detecting the rotor position are proposed continually by researchers. The electrical inductance method based on stator core magnetic saturation effect is presented in [4-8], in which six short time pulses are used to locate and start up the rotor $[4,5]$. This method has advantages of simple hardware circuit design and easy controlling algorithm, whereby the rotor can be started without reversal and can be switched smoothly. The theory introduction, using sensorless BLDCM to determine the rotor position based on the inductance method, is proposed by [7]. But it does not offer the whole experiment process. In [8], the rotor position is estimated by using the change of the voltage of the nonconducting phase. This method is easy to be performed and does not need current sensors, but the precision of the position detection is not satisfied. References $[9,10]$ use state observer and Kalman filtering to detect the motor rotor position. This method has a high degree of dependence on the motor's parameter and a huge calculated amount, which can be realized only by using high-speed DSP. 
Reference [11] introduced a third harmonic detection method based on virtual neutral point electromotive force. This method can obtain accurate commutation signal and is easy to be controlled. But, the position of static rotor cannot be judged by this method, and this method can be realized only by using high speed DSP. Several other control methods are introduced separately in [12-15]. But, all of them just concern the start-up stage after rotor location and ignore the problem about the jitter and reversal of the rotor in the initial stage.

The new start-up method proposed in this paper is as follows: when the motor is static, it can be prelocated within the scope of $\pi / 6$ by using the improved inductance method and supplying power to the coil in a specified direction. In order to know the real rotor position, we should collect the EMF of the nonconducting phase on time and determine the time to start the EMF integrator. Applying the inductance method and the EMF integration to start the initial stage will prevent the motor from jittering and reversing, which results in a smooth start-up process and a rapid response.

\section{Theory Application Analysis}

EMF integration and stator core magnetic saturation effect are cited in this paper as basis of the theory.

2.1. EMF Integration. EMF integration focuses on comparing the EMF integration of the nonconducting phase with a threshold value. When the EMF integration of a phase reaches the threshold value, it is time to commutate this phase. The authors in $[1,14]$ merely provide the demonstration that the EMF integration has no relation to the rotate speed when the rotate speed is constant, but do not take into consideration the condition that the rotate speed is fluctuant in the start-up initial stage.

2.1.1. Demonstration for Independency between EMF Integration and the Rotate Speed in Start-Up Stage. Take a bipolar motor as an example; the instant EMF of the nonconducting phase can be recorded as

$$
e_{\alpha}=N K_{w} \phi w(t)
$$

In this equation, $e_{\alpha}$ is the instant EMF, $N$ is the number of turns of phase winding, $K_{w}$ is the distribution coefficient, $\phi$ is the instant air gap flux, and $\omega(t)$ is the electric angular speed.

At the beginning of the start-up, air gap flux can be approximated as trapezoidal wave. Supposing that the parameter of the motor is constant, the air gap flux merely relates to the angle. Using the $\pi / 6$ electrical angle after EMF zerocrossing point can decide the commutation moment, and the air gap flux reaches the maximum at this moment. Air gap flux can be written as

$$
\phi=\phi_{p} \frac{6 \theta(t)}{\pi}
$$

In this equation, $\theta(t)$ is the rotor angle and $\phi_{p}$ is the flux amplitude of each pole. The EMF integrator begins to work at the moment the EMF crosses zero. The integrator's output voltage is

$$
U=\int_{0}^{t} e_{\alpha} d t
$$

It can be deduced from (1)-(3) that

$$
U=\int_{0}^{t} e_{\alpha} d t=\int_{0}^{t} N K_{w} \phi_{p} \frac{6 \theta(t)}{\pi} w(t) d t .
$$

Based on the timely change of the motor's angle, the angular speed can be written as

$$
w(t)=\frac{d \theta(t)}{d t} .
$$

$$
\begin{aligned}
& \text { If } K_{e}=(6 / \pi) N K_{w} \phi_{p} \text {, it can be deduced from (4)-(5) that } \\
& \qquad \begin{array}{c}
U=\int_{0}^{t} K_{e} \theta(t) \frac{d \theta(t)}{d t} d t \\
U=\int_{0}^{\pi / 6} K_{e} \theta(t) d \theta(t) \\
U=U_{0}=K_{e} \frac{\pi^{2}}{72} .
\end{array}
\end{aligned}
$$

In these equations, $U$ is the integrator's output voltage, $U_{0}$ is the threshold value, and $K_{e}$ is the EMF coefficient. When integrator's output voltage $U$ reaches the threshold value $U_{0}$, the integrator should stop the integration at once and outputs the commutation signal.

2.1.2. Significance of Applying EMF Integration in the StartUp Stage. From the demonstration in Section 2.1.1, it is clear that the value of EMF integration is independent of the rotate speed at the beginning of the start-up. For different motors, the time of commutation can be adjusted by changing the threshold value.

The traditional method needs to set the frequency of commutation before starting. The frequency is decided by the experiment method with the purpose that rotor can reestablish moment balance again when it is disturbed. Because this time cannot be changed in the start-up initial stage, the motor is likely to have commutation error or even start-up failure. Because the value of EMF integration is independent of the rotate speed, the time that reaches one integration value will change according to different loads and constant commutation frequency will also change with it. In this way, the motor can be started smoothly in certain range of load.

In the start-up initial stage, applying EMF integration will also face the condition of inaccurate detection of zerocrossing point, but this will not lead to a serious error in commutation because, in the EMF integration, the result of integration on numerical value is equal to the area that is included by the EMF wave in corresponding integrating range and time axis. From the integration principle and the EMF wave we can see that commutation error will be far less than the zero-crossing point detection error. Taking the EMF rising section of one phase as an example, as shown 


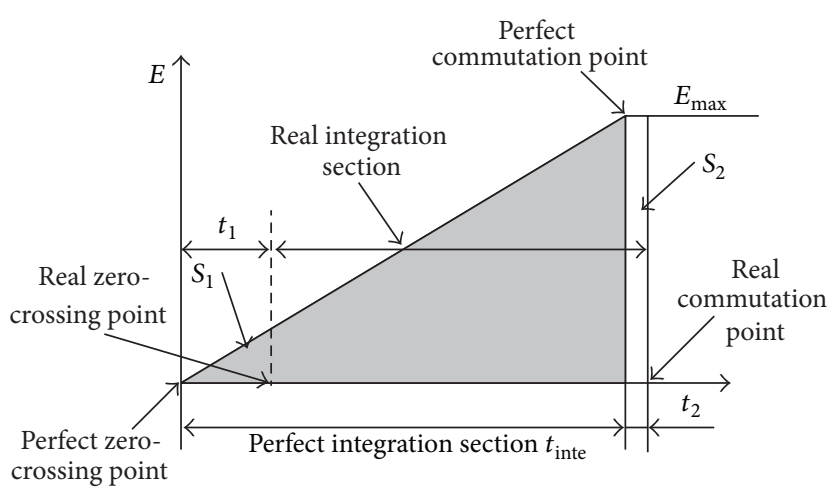

FIGURE 1: Analysis of the error in the method of integrating EMF.

in Figure 1, zero-crossing point detection error is $t_{1}$ and commutation error is $t_{2}$; if we use the scheme of commutating at $\pi / 6$ after EMF zero-crossing point, then $t_{1}=t_{2}$. By contrast, because the integration threshold value is a constant value when adopting EMF integration to commutate, then

$$
\begin{gathered}
S_{1}=S_{2} \\
S_{1}=\frac{1}{2} t_{1} \frac{t_{1}}{t_{\text {inte }}} E_{\max } \\
S_{2}=t_{2} E_{\max } \\
t_{2}=\frac{t_{1}}{2 t_{\text {inte }}} t_{1} .
\end{gathered}
$$

In these equations, $t_{1}$ is the zero-crossing point detection error, $t_{2}$ is the commutation error, $t_{\text {inte }}$ is the perfect integration section, $E_{\max }$ is the maximum EMF, $S_{1}$ is a zero error area, and $S_{2}$ is the commutation delay area.

In the actual operation, $t_{1}$ is far less than $t_{\text {inte }}$ and, as a result, $t_{2}$ is far less than $t_{1}$, which means that zero-crossing point detection error has little effect to the commutation point error.

2.2. Inductance Method Analysis. Using the inductance method to estimate the rotor position is based on the characteristic of the stator core magnetic saturation. In terms of the BLDCM, the flux produced by the stator winding and the permanent magnet flux affect the saturation degree of the stator core together. If the stator core is closer to the pole of the permanent magnet, the magnetization will be strengthened. If the combined flux has an effect to add magnetic force, the more saturate the magnetic field is, the less the winding inductance is and the larger the current is. If the combined flux has an effect to reduce magnetic force, the saturation degree of the magnetic field becomes less, the winding inductance gets larger, and the current becomes smaller.

In the inductance method, the time of duration of the voltage vector is an important parameter, because this method is exactly estimated based on the current response of stator winding. The current response relies on the time constant of the stator winding, and time constant $\tau$ can be

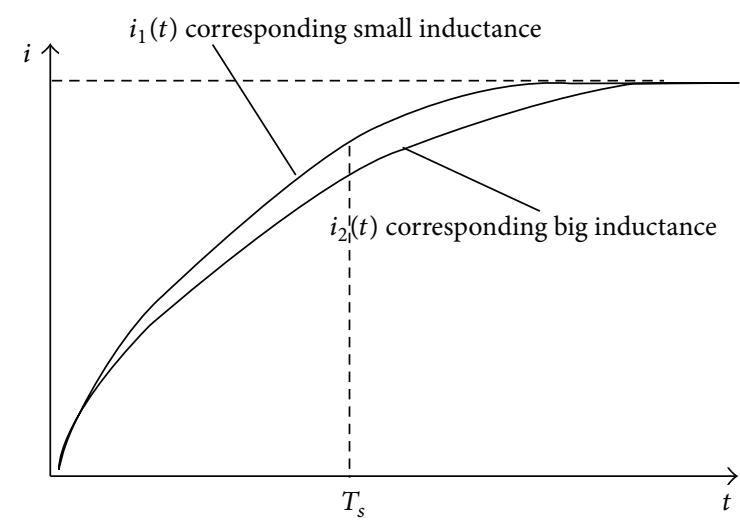

Figure 2: Current response curve.

expressed as $\tau=L / R$. From the equation, time constant is proportional to the inductance.

Figure 2 shows the response curve of current $i_{1}$ and $i_{2}$ when the motor rotor is in different positions, and $i_{1}$ and $i_{2}$ can be calculated as follows:

$$
\begin{aligned}
& i_{1}(t)=\frac{V_{\mathrm{DC}}}{R_{\mathrm{eq}}}\left(1-e^{-\left(t / \tau_{1}\right)}\right) \\
& i_{2}(t)=\frac{V_{\mathrm{DC}}}{R_{\mathrm{eq}}}\left(1-e^{-\left(t / \tau_{2}\right)}\right) .
\end{aligned}
$$

From these equations, $V_{\mathrm{DC}}$ is the DC side voltage, $R_{\mathrm{eq}}$ is the equivalent resistance, and $\tau_{1}$ and $\tau_{2}$ are the time constants of equivalent circuit when the voltage is applied. As we can see, the bigger the time constant is, the smaller the current is. Because the time constant is small, the conduction time should be the one when the difference of current is the most obvious under a different voltage vector.

The current differential $\Delta_{i}$ of $i_{1}$ and $i_{2}$ can be calculated as follows in this way:

$$
\Delta i(t)=i_{2}(t)-i_{1}(t)=\frac{V_{\mathrm{DC}}}{R_{\mathrm{eq}}}\left(e^{-\left(t / \tau_{1}\right)}-e^{-\left(t / \tau_{2}\right)}\right) .
$$

Because the time constant of the motor is small, it can be expressed as $\tau_{2}=\tau_{1}+\Delta, \Delta \approx 0$. Take the derivative of (9) with respect to time, and the point when the difference of the current is the biggest can be found. In terms of each voltage vector, conduction time can be expressed as

$$
T_{s}=\lim _{\Delta \rightarrow 0} \frac{\ln \left(\tau_{1} /\left(\tau_{1}+\Delta\right)\right)}{\left(1 /\left(\tau_{1}+\Delta\right)\right)-\left(1 / \tau_{1}\right)}=\tau_{1} .
$$

From the above, we can see that the preferable conduction time of voltage vector should be the numerical value similar to the time constant of the motor. But, if the time constant of the motor is too big, it can bring about a long conduction time that will force the motor to run. So, reasonable conduction time should be chosen to ensure the accuracy of preposition.

\section{New Method for Start-Up}

The inductance method can detect the rotor position in advance when the motor is static. As power is supplied to 
calculated phases, this method can avoid the consequence of reversal and jitter which can affect the start-up accuracy of the motor. EMF integration can reflect the real angle of the rotor and help the motor start successfully with load. This paper is based on the two advantages above. Firstly, judge the rotor position based on inductance method and determine the integrator start-up time according to the rotor position, and then adjust the commutation frequency to smooth the startup process and eliminate jitter. By using this method, rapid response can also be achieved. Due to the simple arithmetic, those functions can all be realized by common MCU.

3.1. Identify the Initial Rotor Position. Conventional inductance method adopts two-to-two or three-to-three conducting methods, applying six-voltage vector in different direction continually and locating the rotor position in the scope of $\pi / 3$. When using the traditional method, there are two problems: one is that the rotor position cannot be determined precisely, the other is that applying six-voltage vectors at the same time will make the arbitration rules complicated and the programming code lengthy. The improved inductance method is adopted in this paper, which can determine the rotor position in the scope of $\pi / 6$ easily and has simple arbitration rules. It can be linked up with the EMF integration when it starts. The advantage of EMF integration will be more obvious in this way. Sampling quantity and calculated amount decrease and stability increases, which will make the improved effect of start-up more obvious.

The improved inductance method proposed in this paper is as follows: when the motor is static, utilize two-to-two conducting method to apply voltage pulse to the MOSFET. Assuming that the rotor position is shown in Figure 3, firstly apply two-voltage vectors $V_{\mathrm{AB}}$ (direction $C$ ) and $V_{\mathrm{BA}}$ (direction $Z$ ) in the opposite direction for a while. $I_{2}$ and $I_{5}$ are collected and stored as bus current separately. Because the magnetic saturation of the iron core is different, the sizes of $I_{2}$ and $I_{5}$ are also different. If $I_{2}>I_{5}$, from the analysis above, it can be known that the $N$ pole of the rotor is on the left side of line I-II. The rotor is located in the scope of $\pi$ for the first time. Then apply two-voltage vectors $V_{\mathrm{CB}}$ (direction $X$ ) and $V_{\mathrm{AC}}$ (direction $Y$ ) for a while and collect bus currents which can be stored as $I_{3}$ and $I_{1}$.

According to the arbitration rules in this paper, the rotor area can be judged in different conditions. The arbitration rules are as follows: if $I_{2}-I_{1}>0, a=1$ or $a=0$. If $I_{2}-I_{3}>0$, $b=1$ or $b=0$. Bring the results into the arbitration formula $y=2 a+b$. If $y=1$, the rotor is in area 1. If $y=3$, the rotor is in area 2. If $y=2$, the rotor is in area 3 . The rotor position in the right side of I-II can be judged in the same way. In this way, the rotor position can be determined in the range of $\pi / 3$.

According to the assumption above, the rotor should be in area 1. According to the result of location, apply $V_{\mathrm{BC}}$ again and collect bus current and store it as $I_{6}$. Now, there are three positions that the rotor may be in: $Y$ coordinate or a position shift from the $Y$ coordinate by a distance within the range of $\pi / 6$. The method in this paper is treated as the same condition of the rotor in $Y$ coordinate or above the $Y$ coordinate within the range $\pi / 6$. Comparing $I_{6}$ with $I_{2}$, the rotor position can be determined in the scope of $\pi / 6$. According to the assumption

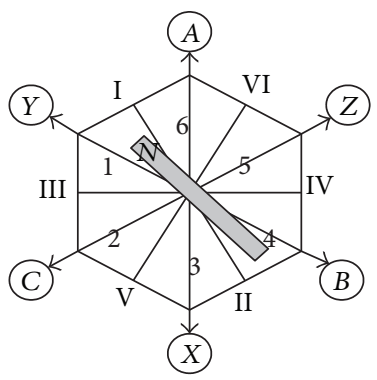

FIgURE 3: The stator magnetomotive force (MMF) and rotor position.

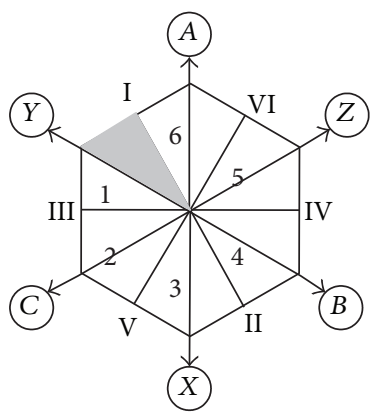

(a)

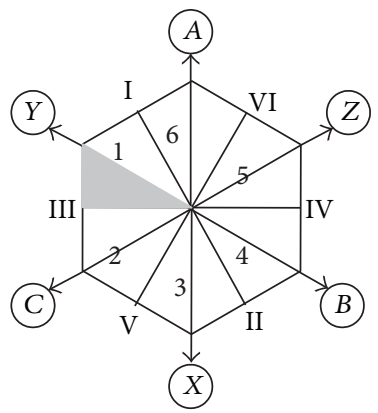

(b)
FIGURE 4: Rotor position.

above, the result now should be $I_{2}<I_{6}$ and the $N$ pole of the rotor should be above the $Y$ coordinate within the range of $\pi / 6$. The judgment of the position of other rotor is similar.

3.2. EMF Integration Start-Up. According to the above, the rotor position is already determined in the range of $\pi / 6$ and the voltage vector can be applied to the rotor according to the torque maximized and predefined rotate direction. Two conditions will come across when the voltage vector is applied: the first is the EMF of nonconducting phase that has already crossed zero, the second is that EMF of nonconducting phase that has not crossed zero.

If the rotor position is in the shadow area in Figure 4(a) now, the power is supplied towards $Z$ direction, and the rotor position has not crossed zero, so the EMF of $C$ phase should be collected. When the voltage vector is $V_{\mathrm{BA}}$, the initial EMF of $C$ phase is minus. The electrical direction should be constant at this moment until the $C$ phase EMF appears zerocrossing point. This zero-crossing point is the common commutation zero-crossing point. Because of the undetermined load, in order to avoid the condition where commutation is too rapid and the rotor rotates in reverse with jitter because of the heavy load, the integrator should be started at the moment when the zero-crossing point is detected. The result of the integration is compared with the integration threshold value on time. If it overflows, the motor will be commutated at once and proceed into the acceleration process.

If the rotor position is in the shadow area in Figure 4(b) now and the power is supplied in A direction by utilizing two-to-two conducting method, now the rotor position is 


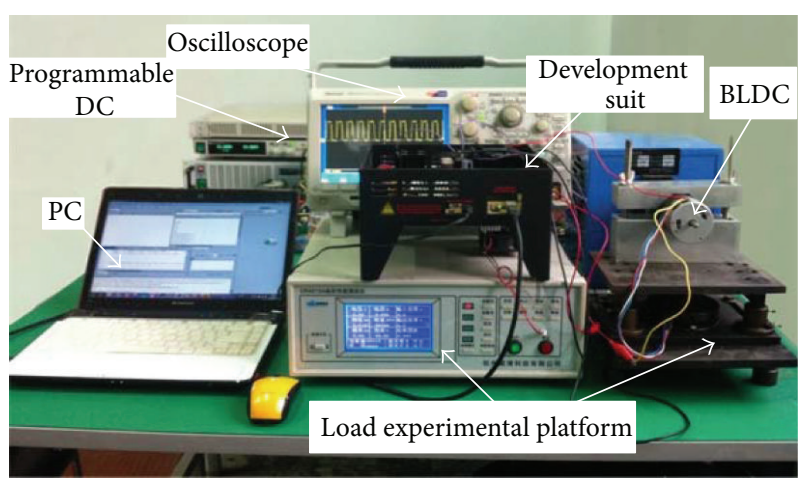

Figure 5: Test bench.

between the zero-crossing point and commutation point. The integrator should be started with the initiator. The result of integration is compared with the integration threshold value on time. If it overflows, the motor will be commutated at once and proceed into the acceleration process.

The commutation error is smaller than the integration initial error, which has been demonstrated as above. As a result of it, the accuracy of zero-crossing point detection should not be worried about. When the integrator is detected to overflow and commutate, the frequency of energization and the speed of the rotor are in synchronization. The motor begins to be accelerated smoothly.

\section{Experimental Verification}

The 32-bit DSP 28335 is selected as the core to build an experimental platform to demonstrate the superiority of the start-up method proposed in this paper. The experimental platform is shown in Figure 5. The rated speed of the motor is $5000 \mathrm{r} / \mathrm{min}$. The experiment is divided into two steps: one is adding inertia load by adopting inertia disc, the other is adding static load on the load test bed.

4.1. Start-Up Method Application Example. In the scope of $\pi / 6$ above $Y$ coordinate, the current waveform and terminal voltage waveform of the rotor detected by the method proposed in this paper are shown in Figure 6.

As shown in the Figure 6(a), $I_{2}>I_{5}, I_{2}-I_{3}>0, I_{2}-I_{1}<0$, we can know that the $N$ pole of the rotor is in the location 1 , according to the arbitration rules proposed in this paper. Because $I_{6}>I_{2}$, the rotor should be above the $Y$ coordinate in the range of $\pi / 6$. After applying the voltage vector, the integrator is started after $C$ EMF crosses zero. A terminal voltage waveform can be obtained as shown in Figure 6(b).

The current waveform of the rotor in the scope of $\pi / 6$ below $Y$ coordinate obtained by the method of this paper is shown in Figure 7, and the start-up terminal voltage waveform on the load test bed without load at this moment is also shown in it.

As shown in the Figure $7(\mathrm{a}), I_{2}>I_{5}, I_{2}-I_{3}>0, I_{2}-I_{1}<0$, we can know that the $N$ pole of the rotor is in the location 1 , according to the arbitration rules proposed in this paper. And, because $I_{6}<I_{2}$, the rotor should be in the range of $\pi / 6$ below the $Y$ coordinate. Start the integrator when applying

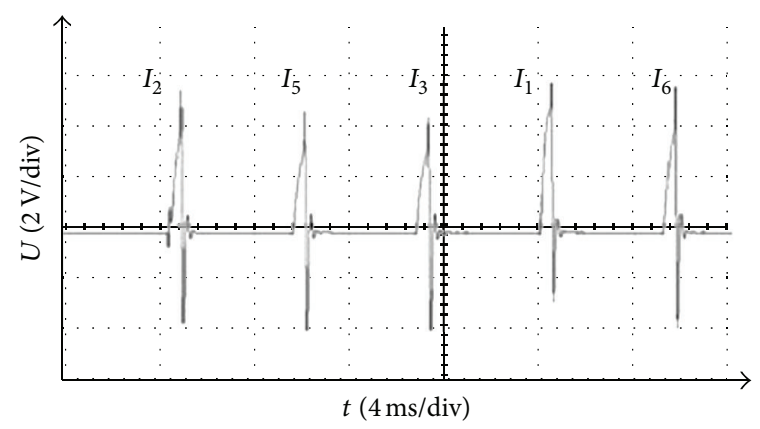

(a) Current response waveform

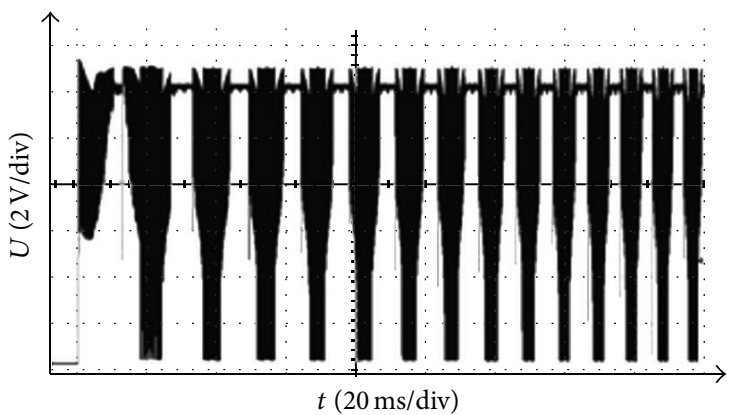

(b) End voltage waveform

FIGURE 6: End voltage waveform and currents response under EMF non-zero crossing.

the voltage vector $V_{\mathrm{BC}}$, and terminal voltage waveform can be obtained as shown in Figure 7(b).

The rotor position can be known in advance by utilizing method proposed in this paper. The method can ensure that the start-up process has no reversal, no jitter, and commutation signal, and rotor synchronized quickly to get a better end voltage waveform when it starts.

4.2. Contrast Test. The advantage of the method proposed in this paper is that the rotor position can be determined in advance and a different start-up program can be executed according to the rotor position. Some problems such as jitter and reversal of the motor are avoided. The application of integration can also make the motor achieve a nice startup effect with heavy or light load. The applied range of the motor becomes wider and its start-up process becomes better. Compared with the start-up waveform obtained by the traditional method with different load, the advantage of the method proposed in this paper can be demonstrated.

The experimental results under inertia disk with the traditional method and the method proposed in this paper are shown in Figure 8. Comparing the two figures, it can be seen that when adding the inertia load, a severe jitter appears in the start-up stage using the traditional method and the smooth start-up of the motor is not realized in the commutation period displayed by the figure. The method of this paper determines the rotor position in the first period and it starts according to the actual load condition, which can make the start-up process more smooth without jitter.

Terminal voltage waveform with no load, rated torque, and twice the rated torque experimented on the load test bed 


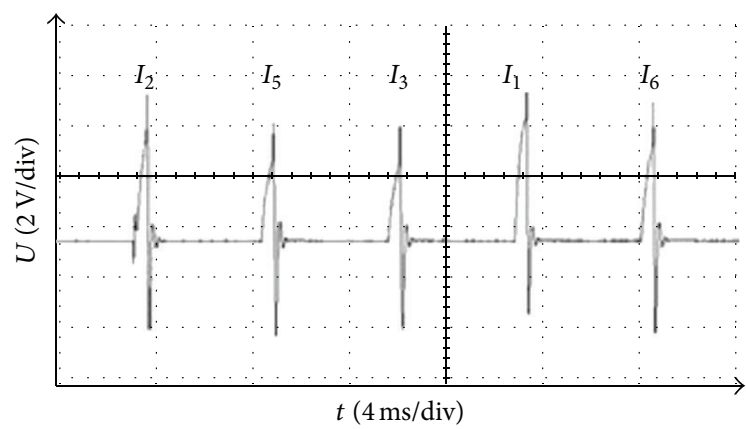

(a) Current waveform

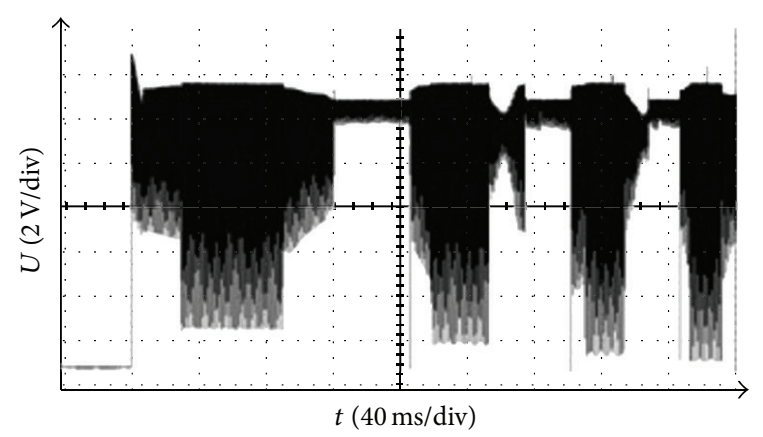

(b) End voltage waveform

FIGURE 7: Terminal voltage waveform and currents response under EMF zero crossing.

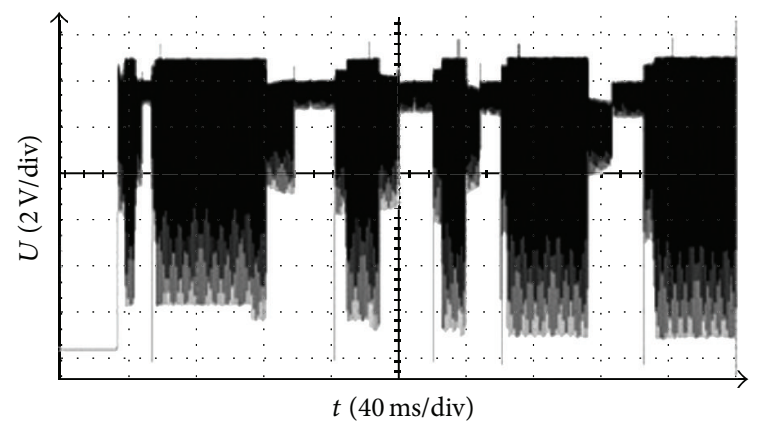

(a) The traditional method

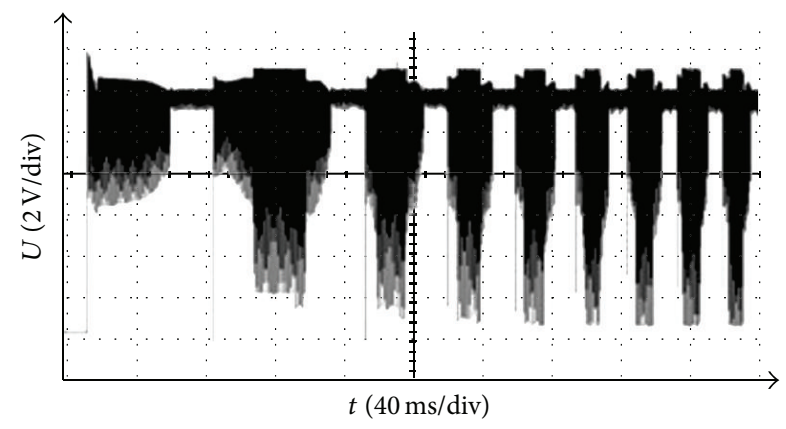

(b) The method proposed in this paper

Figure 8: End voltage waveform with two different methods under inertia disk.

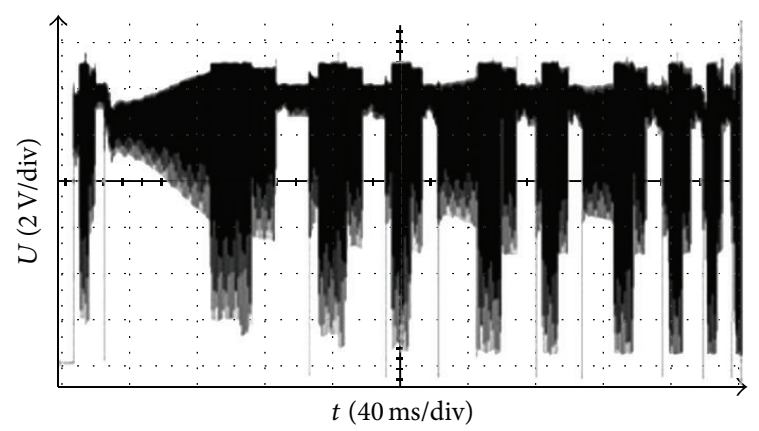

(a) The traditional method

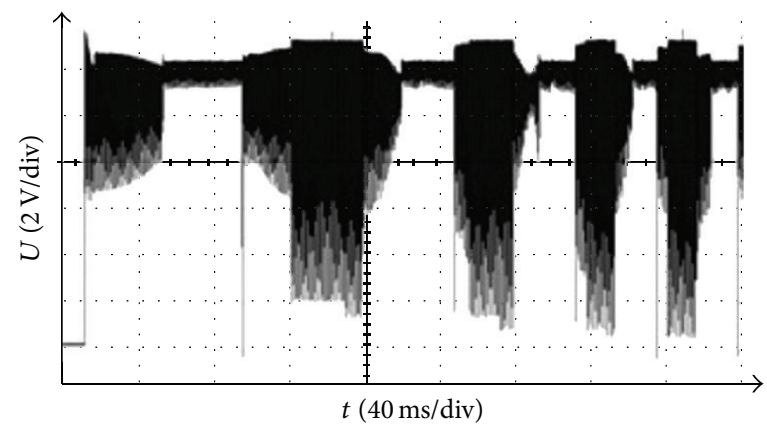

(b) The method proposed in this paper

FIGURE 9: Terminal voltage waveform with the traditional method and the method proposed in this paper under load.

with the traditional method and the method of this paper are shown in Figures 9, 10, and 11.

From the comparison of the three figures above, the motor performance that use traditional method has serious fluctuation and bad waveform both in no-load, fractional load, and overload. When controlling with the method proposed in this paper, the commutation signal and the rotor can be synchronized in the first commutation period, whether with fractional load or overload. And, the fluctuation is small and the start-up is smooth. From the figures above, the integration time changes with the load can be easily found.

Current waveforms obtained by two different start-up methods of the motor are shown in Figure 12.

As can be seen from the results above, the method proposed in this paper has an obvious advantage over the traditional method. Firstly, two sets of waveforms of the current and phase terminal voltage applying the method of this paper are shown in the example, while the rotor position in the beginning of the start is different. It shows that the inductance method can detect the position of the rotor fast and accurately and then judge when to apply the EMF. This can ensure that the process starts smoothly and steady, and also ensure that the motor commutes precisely. Experiments of the different rotor position in the start have been carried out to verify the reliability of this method. The results indicate that the method is credible. In the following contrast tests, the motor is started under different load. The comparison waveforms of phase terminal voltage show clearly that this method is superior 


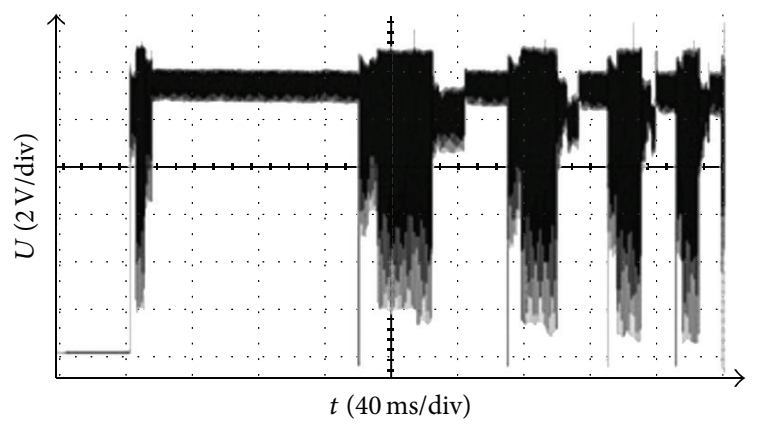

(a) The traditional method

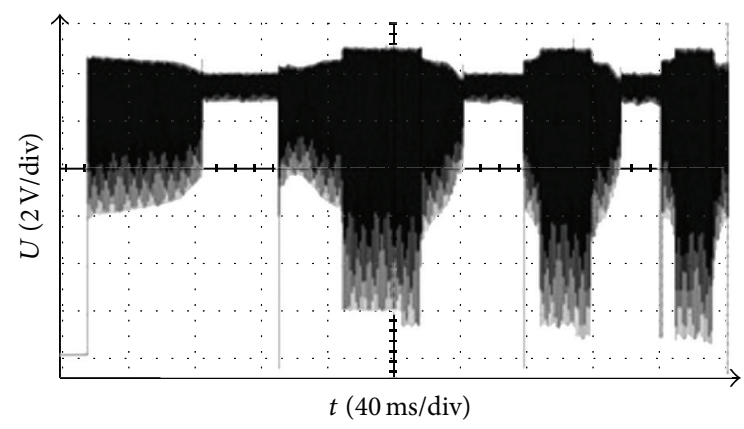

(b) The method proposed in this paper

FIgURE 10: The end voltage waveform with the traditional method and the method proposed in this paper with rated torque.

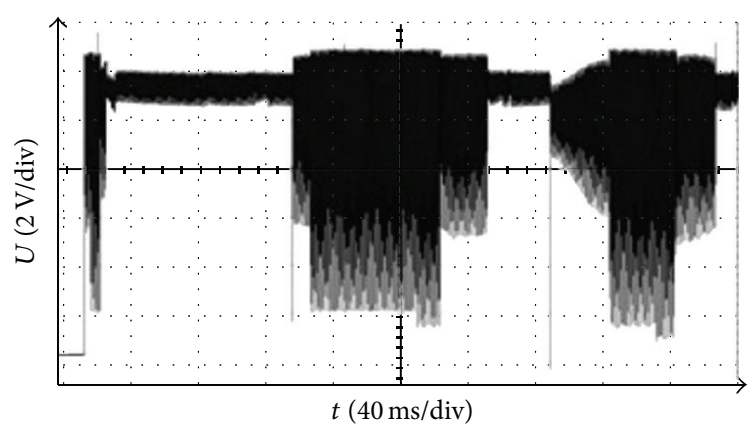

(a) The traditional method

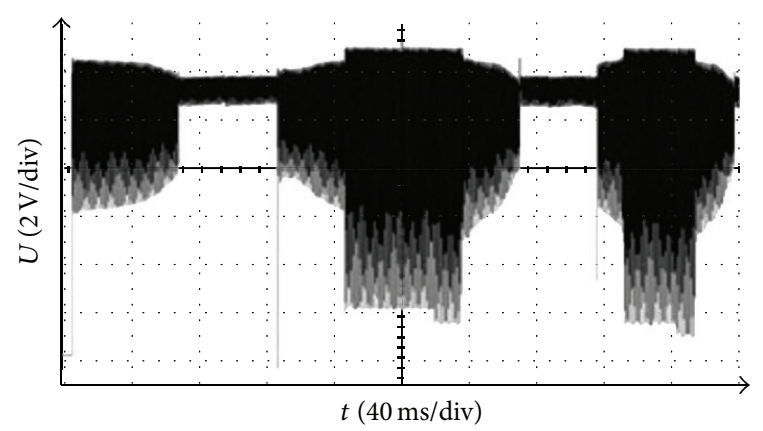

(b) The method proposed in this paper

FIGURE 11: End voltage waveform with traditional method and method proposed in this paper under twice the rated torque.

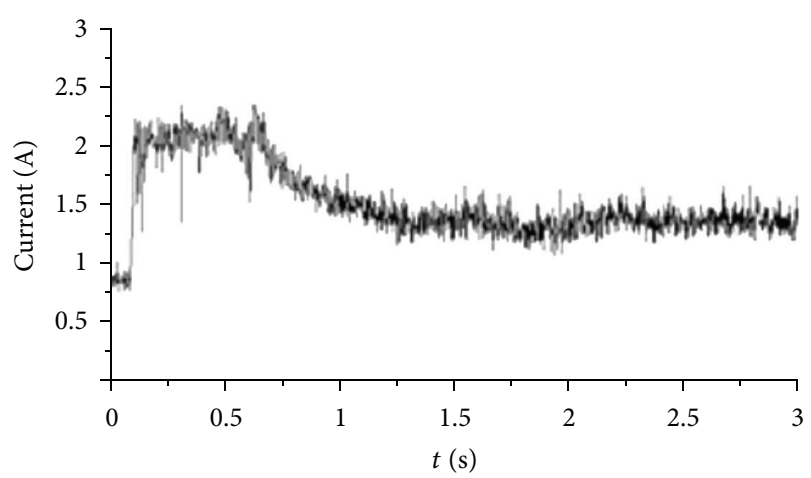

(a) The traditional method

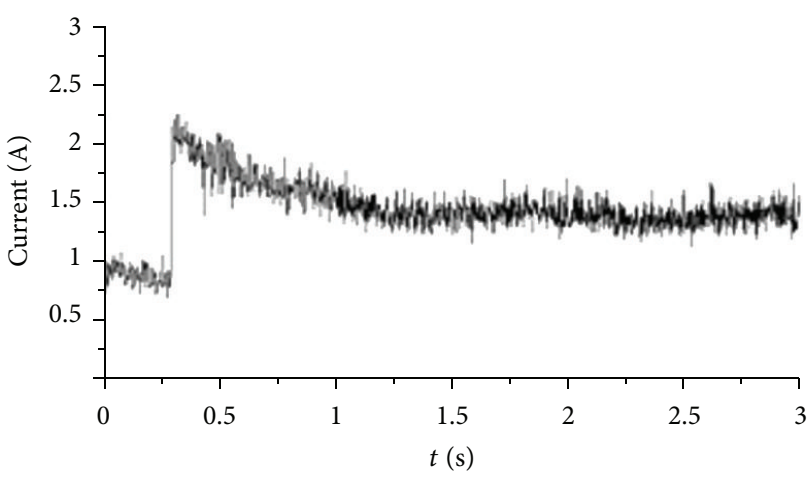

(b) The method proposed in this paper

FIGURE 12: Comparison of the current of two different methods.

to the traditional method and the starting process is smooth and fast. The results demonstrate that this method can guarantee the startability when the motor is under different load. Due to the improved performance, this method can expand the application range of sensorless BLDC. At last, two sets of current waveform are given to show the improvement of this method. It can be seen from the figure, that this method can make the current achieve steadily fast and smoothly without distinct jitter. The comparison of the current waveform indicates that this method can alleviate the phenomenon of jitter in the start and smooth the starting process.

\section{Conclusion}

The advantages of the inductance method and the EMF integration are analyzed in detail in this paper. And, after combining these two methods, a new start-up method for sensorless BLDCM is proposed. The integrator start-up time is determined by implementing the improved inductance method and the new arbitration rules. The experiment demonstrates the advantages of the new start-up method proposed in this paper compared to the traditional one in start-up stage. The location of the rotor when the motor is static is realized, and the commutation signal and the rotor position can be synchronized at once. It increases the startup success rate with load and improves rapidity and stability of the current response. 


\section{Conflict of Interests}

The authors declare that there is no conflict of interests regarding the publication of this paper.

\section{Acknowledgments}

The work is sponsored by the Aerospace Support Technology Fund (2013-HT-HGD09), the National Laboratory for Electric Vehicles Foundations (NELEV-2013-004), the Shandong Province Outstanding Young Scientists Research Award Funds (BS2012NJ001), and the Beijing Science and Technology Project (Z121100005612001).

\section{References}

[1] C. Xia, Brushless Direct Current Motor Contrl System, Science Press, Beijing, China, 2009.

[2] J. Shen and X. Lü, "Analysis of 3-step start-upfor sensorless BLDC motor," Small \& Special Electrical Machine, vol. 26, no. 5, pp. 8-11, 1998.

[3] M. Lin, Q. Li, and P. Yang, "Analysis and compensation of rotor position detection error in a position sensorless BLDC motor," Small \& Special Electrical Machines, vol. 31, no. 6, pp. 8-12, 2003.

[4] Z. Zhang, M. Lin, and G. Zhou, "Anti-reverse rotation startup and smoothly switching of sensorless brushless DC motor," Transactions of China Electrotechnical Society, vol. 24, no. 11, pp. 26-32, 2009.

[5] M. Lin, W. Liu, and G. Zhou, "A location and acceleration method of sensorless brushless DC motor utilizing short voltage pulse," Transactions of China Electrotechnical Society, vol. 26, no. 9, pp. 80-86, 2011.

[6] W.-J. Lee and S.-K. Sul, "A new starting method of BLDC motors without position sensor," IEEE Transactions on Industry Applications, vol. 42, no. 6, pp. 1532-1538, 2006.

[7] X. Li, X. Ge, and D. Wu, "Identification of the rotor initial position of brushless DC motor based on inductance method," Small \& Special Electrical Machine, vol. 5, pp. 6-8, 2009.

[8] Q. Wang, Y. Wang, D. Kong et al., "The discrete from of tracking differentiator," Proceeding of the CSEE, vol. 32, no. 33, pp. 105110, 2012.

[9] B. Terzic and M. Jadric, "Design and implementation of the extended Kalman filter for the speed and rotor position estimation of brushless DC motor," IEEE Transactions on Industrial Electronics, vol. 48, no. 6, pp. 1065-1073, 2001.

[10] T.-N. Shi, Q. Zhang, C.-L. Xia, P. Song, and J.-R. Wan, "Estimates of rotor position and velocity of brushless DC motor with UKF algorithm," Journal of Tianjin University Science and Technology, vol. 41, no. 3, pp. 338-343, 2008.

[11] Z.-Y. Sun, G.-B. Li, Q. Hou et al., "Research on digital BLDCM speed-adjusting system based on DSP," Power System Protection and Control, vol. 41, no. 3, pp. 338-343, 2008.

[12] X. Xue and C. Yang, "A unified approach to direct back EMF detection for brushless DC motor," Transactions of China Electrotechnical Society, vol. 25, no. 9, pp. 62-68, 2010.

[13] C.-Y. Zhi, R.-L. Wang, D.-K. Tang, and R.-T. Liu, "Study on fuzzy self-adaptive PID control strategy for brushless DC motor," Power System Protection and Control, vol. 38, no. 8, pp. 35-42, 2010.
[14] S. Li, H. Ma, G. Lu et al., "Sensorless brushless DC motor control based on back-EMF integral compensation method," Small \& Special Electrical Machine, vol. 53, no. 6, pp. 37-39, 2008.

[15] X.-F. Wang, Z.-Y. Du, and X.-D. Wang, "Hybrid control of brushless DC motor based on LS-SVM and sliding mode variable structure," Power System Protection and Control, vol. 39, no. 2, pp. 70-79, 2011. 


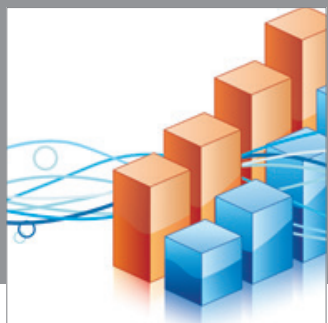

Advances in

Operations Research

mansans

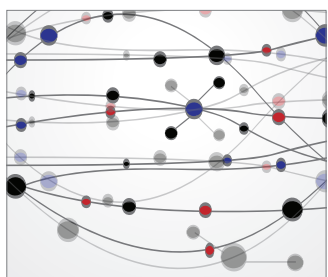

The Scientific World Journal
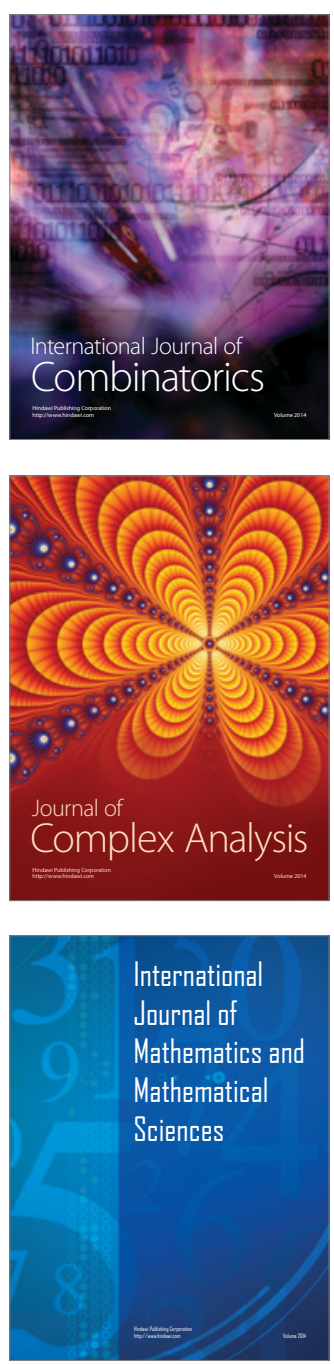
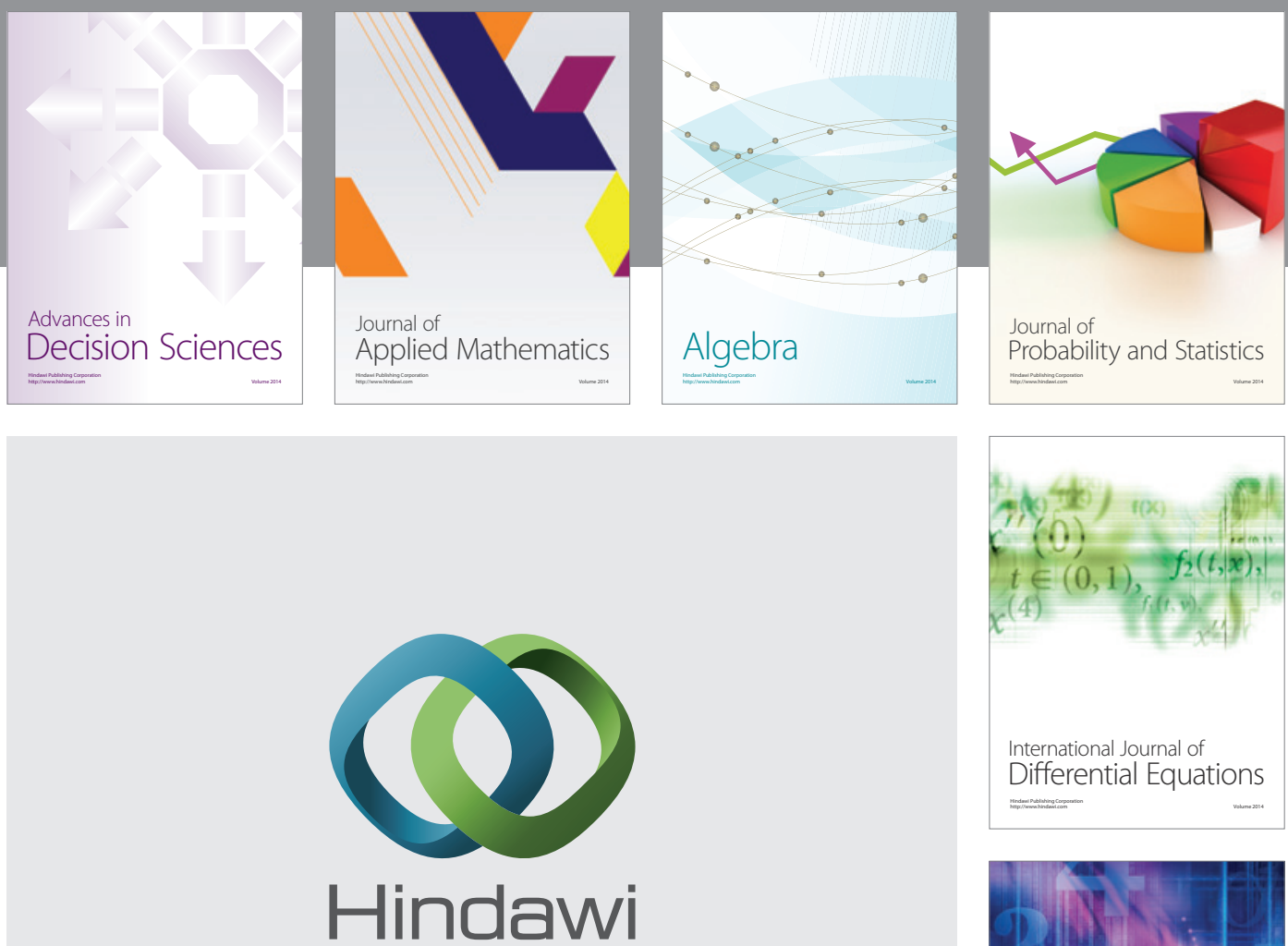

Submit your manuscripts at http://www.hindawi.com
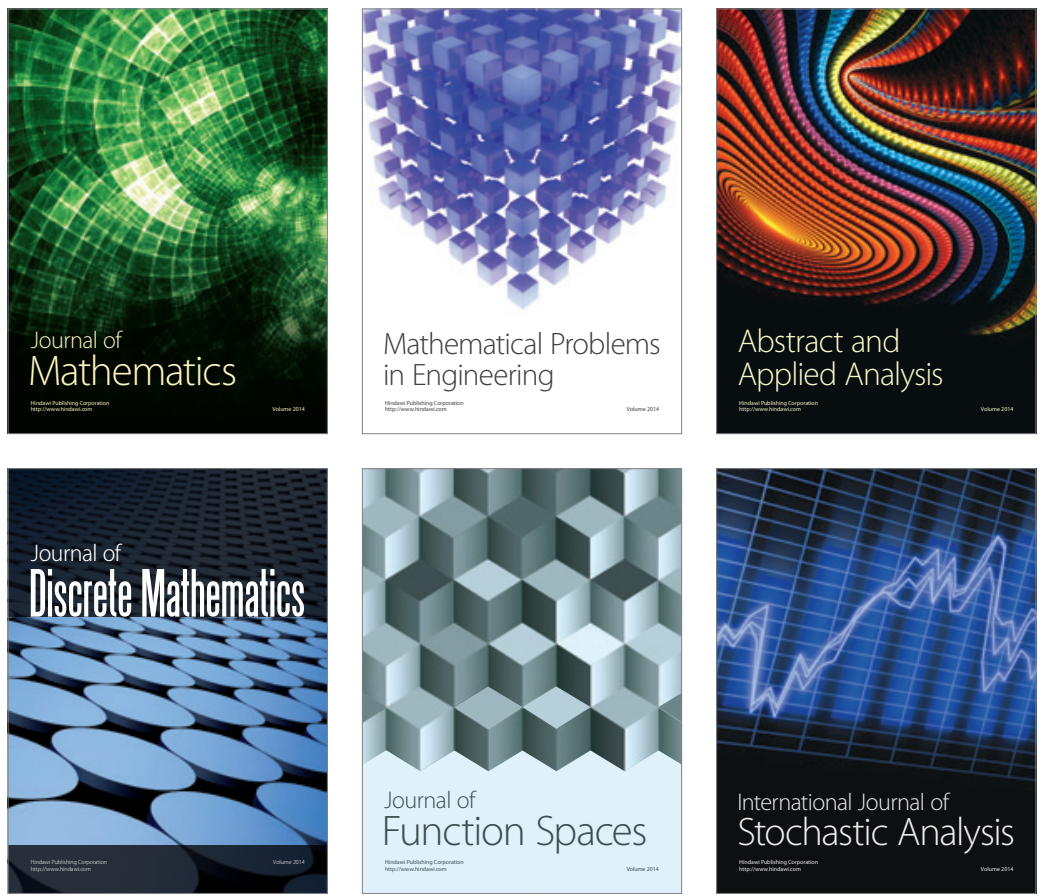

Journal of

Function Spaces

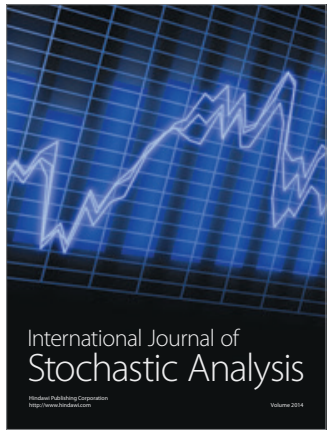

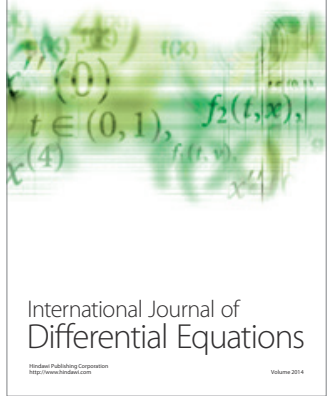
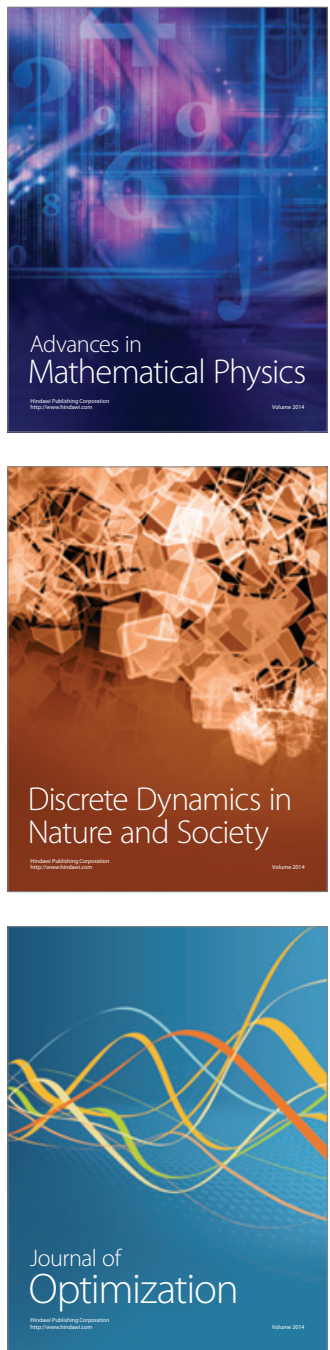